\title{
Board examination for anatomical pathology in Switzerland: two intense days to verify professional competence
}

\author{
Hans-Anton Lehr • Holger Moch • Brigitte Christen • \\ Alexandra Safret • Mathias Gugger • Matthias Rössle • \\ Michael von Gunten • Robert Lemoine • \\ Ann-Marie Kurt • Rosmarie Caduff • Arnold Walter • \\ Gad Singer • Peter Luscieti • Fridolin Bannwart • \\ Claude Y. Genton
}

Received: 4 May 2012 / Revised: 4 June 2012 / Accepted: 5 June 2012 /Published online: 21 June 2012

(C) Springer-Verlag 2012

\begin{abstract}
About 15 years ago, the Swiss Society of Pathology has developed and implemented a board examination in anatomical pathology. We describe herein the contents covered by this 2-day exam (autopsy pathology,
\end{abstract}

\section{H.-A. Lehr · C. Y. Genton}

Institute of Pathology, University of Lausanne,

Lausanne, Switzerland

C. Y. Genton

e-mail: claude.genton@bluewin.ch

H. Moch $\cdot$ M. Rössle $\cdot$ R. Caduff

Institute of Pathology, University of Zurich,

Zurich, Switzerland

H. Moch

e-mail: holger.moch@usz.ch

M. Rössle

e-mail: Matthias.Roessle@usz.ch

R. Caduff

e-mail: rosmarie.caduff@usz.ch

B. Christen · A. Safret

Institute of Pathology ADMED,

Neuchâtel, Switzerland

B. Christen

e-mail: brigitte.christen@ne.ch

\section{A. Safret}

e-mail: alexandra.safret@ne.ch

\section{Gugger}

Institute of Pathology, University of Bern,

Bern, Switzerland

e-mail: mathias.gugger@pathology.unibe.ch

M. von Gunten

Institute of Pathology Länggasse,

Bern, Switzerland

e-mail: mvongunten@patholaenggasse.ch cytology, histopathology, molecular pathology, and basic knowledge about mechanisms of disease) and its exact modalities, sketch a brief history of the exam, and finish with a concise discussion about the possible objectives and

\section{R. Lemoine $\cdot$ A.-M. Kurt}

Institute of Pathology Viollier,

Geneva, Switzerland

R. Lemoine

e-mail: robert.lemoine@viollier.ch

A.-M. Kurt

e-mail: anne-marie.kurt@viollier.ch

A. Walter

Cantonal Hospital of Lucerne,

Lucerne, Switzerland

e-mail: walter.arnold@luks.ch

G. Singer

Cantonal Hospital of Baden,

Baden, Switzerland

e-mail: Gad.Singer@ksb.ch

P. Luscieti

Cantonal Hospital of Locarno,

Locarno, Switzerland

F. Bannwart

Institute of Pathology Medica,

Zürich, Switzerland

e-mai: bannwart@medica-labor.ch

H.-A. Lehr $(\square)$

Institut Universitaire de Pathologie, Centre Hospitalier

Universitaire Vaudois (CHUV),

Rue du Bugnon 25,

1011 Lausanne, Switzerland

e-mail: hansanton.lehr@yahoo.de 
putative benefits weighed against the hardship that it imposes on the candidates.

Keywords Board examination · Training $\cdot$ Anatomical pathology $\cdot$ Quality control

In 1996, the Swiss Society of Pathology (Schweizerische Gesellschaft für Pathologie, SGPath) definitely implemented a national examination to ensure the professional competence of its board members. This exam consists of a 2-day "tour de force" that covers all aspects of the professional activity of an anatomical pathologist. Only senior residents can apply for the exam by writing to the secretary of the president of the board committee, usually 6-12 months in advance, and usually dedicate most of their time to its preparation during their last year of residency. The examination takes place twice a year, sometimes even three times if the number of applicants largely exceeds the capacity of two sessions. Indeed, a maximum of six candidates can be accepted for each exam (seven in rare exceptional situations). The venue of the exam changes each time, according to a rotating system between academic pathology institutions (Basel, Bern, Geneva, Lausanne, and Zürich) and pathology departments in larger cantonal or city hospitals (Baden, Liestal, Lucerne, St. Gall, and Triemli Hospital, Zurich).

The main venue consists of a conference room with six tables on which the candidates find a diagnostic microscope as well as a collection of standard textbooks of diagnostic pathology which can be consulted freely during the slide sessions (Fig. 1). In addition, candidates are free to bring and use their own selection of textbooks for the diagnostic

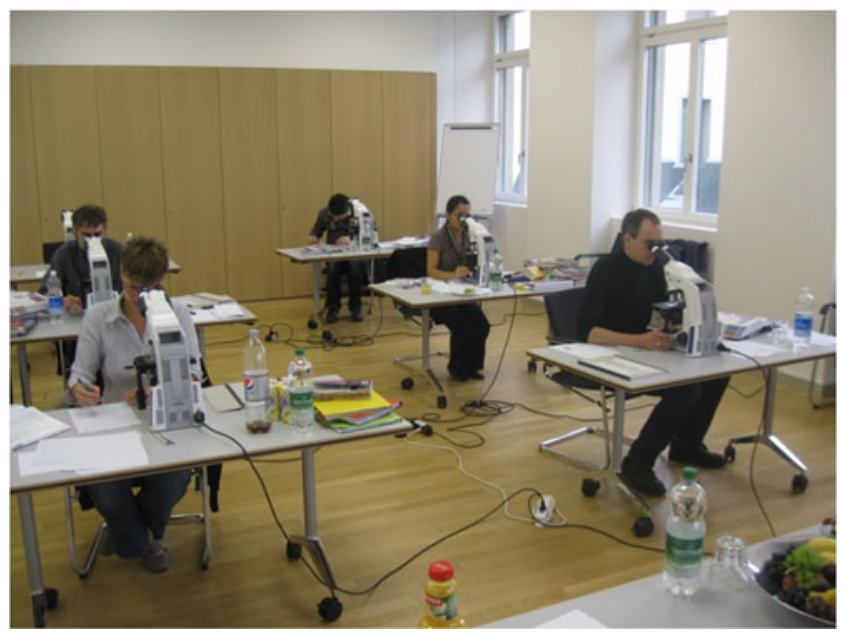

Fig. 1 Candidates dispose of high-quality diagnostic microscopes and their proper sets of slides. The use of standard textbooks is permitted during the parts where slides are examined, but prohibited during the theoretical part with multiple choice and open questions (photo taken at the occasion of the exam in September 2011 in Lucerne, in the rooms of the Academy for Medical Training and Simulation) part of the exam, but on-line consultation of web sites is not allowed. In addition, a room with a multihead (three heads minimum) microscope has to be made available by the hosting institution for the discussion of slides between candidates and members of the exam committee. Finally, the institution that hosts the exam must assure that the candidates can examine the unfixed organs of a recent autopsy, usually in the autopsy suite (Fig. 2).

As a rule, the examination begins on a Thursday afternoon at one o'clock and ends the following day around four o'clock. Knowledge in cytopathology is first examined. For 1 hour, ten cases of gynecological and non-gynecological exfoliative and aspiration cytology are rotated between the candidates in such a way that each candidate has at least 4 minutes to examine each slide, with the possible option of reviewing selected slides once again if so wished. The best diagnosis must be chosen among five possible diagnoses in a multiple choice modus. The candidates are free to consult textbooks, just as they would do during their daily diagnostic activity. In addition, two free text questions about cytopathology have to be answered by the candidates, without the help of textbooks.

The second part of the examination covers autopsy pathology. For 3 hours, the candidates have to analyze and describe the pathological findings of three autopsy cases and formulate a final diagnostic conclusion that also integrates the pertinent clinical information. Two of the three autopsy cases are based on collections of slides (10-16 slides of the major organs), accompanied by a description of the macroscopic findings and relevant clinical information, including specific questions raised by the clinicians. For that purpose, all candidates receive one of six identical

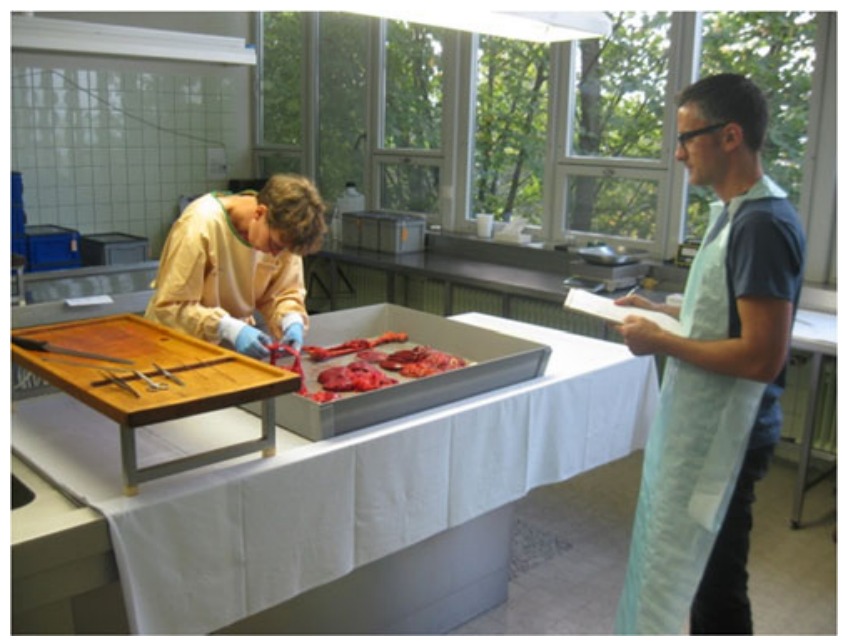

Fig. 2 Autopsy exam: Candidates are asked to examine the fresh organs of a recent autopsy usually in the autopsy suite of the hosting institute and describe their macroscopic impressions, which are being transcribed by one of the examiners (photo taken at the occasion of the exam in September 2011 in Lucerne, autopsy suite of the Cantonal Institute of Pathology) 
sets of slides. The third autopsy case is based on the examination of the fresh organs of a recent autopsy which are to be viewed in the local autopsy suite - along with a copy of the available clinical information and a list of specific questions to be addressed by the autopsy. For practical purposes, each of the six candidates is individually taken to the autopsy suite by two members of the exam committee. The candidate has 20 minutes to examine the organs and dictate his/her observations to one of the committee members (preferentially the one with the most readable handwriting!; Fig. 2) The candidate then returns to the examination room and writes up his/her observations, diagnoses, and final conclusions. For this part of the exam, textbooks can be freely used by the candidates. The autopsy exam terminates the first day of the examination.

The next morning starts at 8 o'clock with a 1 hour exam dedicated to macroscopic pathology. The candidates find a stack of 12 letter size high-quality color photos of "typical" macroscopic presentations of lesions in either surgical resection specimens or organs from autopsies. They have to identify the lesion, either by naming the pathological process/diagnosis (free text) or by selecting their best solution from a list of five possibilities (multiple choice). In addition, the candidates are asked to (a) draw on the illustration of the specimen in which regions they would select tissue blocks for the subsequent histological analysis and (b) indicate which auxiliary tests (i.e., special stains, immunohistochemistry, molecular pathology, other) they would like to perform in order to confirm, and/or to complement their diagnosis.

After a short pause follows a 3-hour period, during which candidates have to examine 24 histological slides and name the correct diagnosis. In the case that they are unable to come to a definite diagnosis, they have to list their differential diagnoses and state the approach they would choose to arrive at a final diagnosis (i.e., special stains, immunohistochemistry, molecular pathology, etc.). As for the cytopathology and the autopsy parts, candidates are free to use textbooks for this period, just as they would do during their daily diagnostic practice.

After the lunch break comes the theoretical part, in which 12 multiple choice and three of four free text questions have to be answered. This part of the exam is primarily intended to test the knowledge of the candidates in terms of pathophysiological concepts and novel techniques as they pertain to diagnostic anatomical pathology, but also in terms of scientific (for example: what do you have to consider when planning a biomarker study) and legal/ethical concepts (i.e., professional secret in clin-path conferences and research projects, use of human tissues for research and teaching, autopsy regulations, certification/accreditation of a diagnostic service, etc.). The candidates are asked to respond to at least three of the four free text questions, the fourth may be ignored. For this part of the examination, the use of textbooks is no longer permitted. This part of the exam also integrates an increasing number of questions related to concepts and techniques of molecular pathology.

The board examination comes to its end with a $15-30$ minutes interview of each candidate separately with two members of the exam committee, around a multihead microscope. During this interview, the candidate gets a chance to review some of the cytology and histology slides that he/she had failed to correctly identify during the test, explain his/her initial line of reasoning, and propose a better solution. The members of the committee find this interview particularly helpful in getting a reliable feeling of the background knowledge of the candidates. As the distribution of points from the different parts of the exam shows (Table 1), it becomes apparent that this interview may be particularly helpful in all cases of "shaky" candidates, who have not been able, up to this point, to secure enough points in the preceding parts of the exam for a clear pass. It also gives the members of the committee quite a good idea of where the candidate stands in his/her formation, whether he/she has had so far missed out on certain aspects of his/her training (i.e., the cytology rotation), notions that will eventually help to arrive at a final
Table 1 Algorithm for point assignment for final "pass" score

\begin{tabular}{lllll}
\hline Time & Test & Consisting of & Points per correct answer & Maximum \\
\hline $1 \mathrm{~h}$ & Cytology & 10 slides, 2 questions & 2 points per case/question & 24 points \\
$3 \mathrm{~h}^{\mathrm{a}}$ & Autopsy I & Autopsy, fresh organs & Case-specific distribution & 16 points \\
& Autopsy II & Autopsy, slides & Case-specific distribution & 16 points \\
& Autopsy III & Autopsy, slides & Case-specific distribution & 16 points \\
$1 \mathrm{~h}$ & Macro & 12 specimen images & 2 points per case & 24 points \\
$3 \mathrm{~h}$ & Histology & 24 slides $^{\mathrm{b}}$ & 4 points per case & 96 points \\
$1 \mathrm{~h}^{\mathrm{a}}$ & Theory I & 12 MC questions $^{\mathrm{a}}$ & 2 points per case & 24 points \\
& Theory II & 3 free questions & 4 points per question & 12 points \\
To-30 min & Interview & Multihead microscope & Individual & 12 points \\
Total & & & & 240 points \\
Points needed to pass $(75 \%)$ & & & 180 points \\
\hline
\end{tabular}


decision, and to council the candidate. Based on the results of the candidates in the different parts of the test, scores are assigned according to a pre-defined algorithm which is presented in Table 1.

Compared to similar tests in the USA and various European countries, the Swiss exam lasts certainly longer (2 days) and covers a wider field (cytology + autopsy + histology + macroscopy + theory). While this may be considered as a disadvantage, adding stress and hardship on the candidates who have to organize their lives (leave of absence, babysitter for children, etc.) and stay overnight in a foreign town, it does make sure that no one fails the exam simply because of bad chance with too few and over-focused questions. In that respect, the exam is designed to prevent false-negative results, i.e., failing a candidate who is competent, and to prevent falsepositive results, i.e., allowing an unprepared candidate to pass unnoticed [1].

The questions and cases selected for the exam reflect the constant effort to recreate as closely as possible the realworld situation of the pathologists during their daily work dealing with a wide spectrum of simple, more challenging, and also some quite difficult cases, i.e., when they have to council clinicians about rare diagnoses and novel diagnostic techniques, and not to forget that they should be able to contribute also to academic activities. It is hence not surprising that the last few years have seen a gradual increase of questions that pertain to molecular pathology and its role in predicting the response of novel targeted treatments in an increasing number of malignant tumors. In this respect, the contents of the examination constantly adapt to changes in the postgraduate curriculum in medicine and anatomical pathology, respond for instance to a dwindling number of autopsies on one side, and an increased focus on molecular pathology on the other side. These modifications of the curriculum are currently being discussed and prepared in detail at the level of the respective professional societies and legal instances, and the exam will be modified accordingly.

History The initiative to create a professional exam in anatomical pathology goes back to the decision of the Swiss Society of Pathology (SSPath/SGPath) in the early 1990s. The scheme of the examination was first tested in 1993 with six candidates who participated on a purely voluntary basis (one of the candidates became father on the day of the exam, which was considered a positive omen for the project). In the first few years, unofficial diplomas were handed out to the candidates, stating that the candidates had passed the board examination in anatomical pathology. From 1994 on, the participation at the examination but not its passing became compulsory and only since 1996 did the successful outcome of the exam become compulsory for board certification. This was also the time when the exam was accredited by the Swiss Medical Association, Federatio Medicorum Helveticorum
(FMH, the national institution that certifies medical specialties). It was only after intense discussions between members of the SSPath/SGPath and the FMH that this official institution acknowledged and accredited the board examination in anatomical pathology — and at the same time saluted the quality of the exam, its broad spectrum and the initiative of SSPath/SGPath members for mounting this exam. Today, FMH and SSPath/SGPath collaborate harmoniously in the certification of anatomical pathologists. The FMH verifies whether the candidates can document the necessary prerequisites for the board certification (i.e., a rotation through different diagnostic services, log book documenting the required minimum numbers of autopsies, histology and cytology cases, as well as frozen sections, participation in conferences and slide seminars, authorship in scientific publications, etc.). The SSPath/SGPath administers the board examination and issues a certificate of successful participation, without which no board certification will be ultimately issued by the FMH.

During the first years, the candidates also had to realize a frozen section (cutting, staining, and interpreting). In 1999, the cytology part was added to the exam and a few years later the discussion between candidates and examiners at a multihead microscope. Over the years, failure rates have been low $(5-10 \%)$. Candidates who had failed had the chance to take the exam again, usually 6 months or 1 year after the initial exam, and so far everyone who had failed once eventually passed in the next exam for which he/she sat. Even though this has never happened in the history of the exam, the candidates are free to take the exam yet again in case they failed a second time. During the first years of the exam, a large collection of slides had been put together, and even though the collection was quite extensive, covering all aspects of anatomical pathology, candidates eventually realized that some of the cases/ questions recurred and started to generate memory protocols that were then passed on to the "next generation" of candidates. This in turn did not escape the members of the exam committee who noticed that many of the more difficult cases were easily answered - if the cases had been used in an exam before. A graphic rendition of points reached in the different parts of the exam underlines this fact, where between 1999 and 2009 there was a steady and statistically significant increment of the points obtained on histological slides, while at the same time results in the autopsy and the cytology parts (which were renewed in every exam) remained at a rather constant level (Fig. 3). When in 2009 the decision was made to no longer reuse slides from the collection, the results obtained on histological slides fell to levels similar to those before the widespread distribution of "exam diagnoses." The simultaneous drop in the results obtained in the theory part of the exam reflects a change in the orientation of theoretical questions, with an increasing emphasis being placed on practical aspects of diagnostic molecular pathology-in agreement with the new spirit of the boards of anatomical pathology set 
Fig. 3 Graphic rendition of points obtained by the candidates in the different parts of the exam over the past 12 years. Note that there was a significant increase from 1999 to 2009 of the results obtained in the histology part (upper left panel), likely due to the "tradition" of diagnoses of exam slides from one generation of candidates to the next. No such trend was observed for those parts where no "old cases" (cytology and autopsy) were being used. Note also that the results returned to "baseline" when the decision was made in 2009 to not reuse slides and theoretical questions from the exam collection that had been used in prior exams
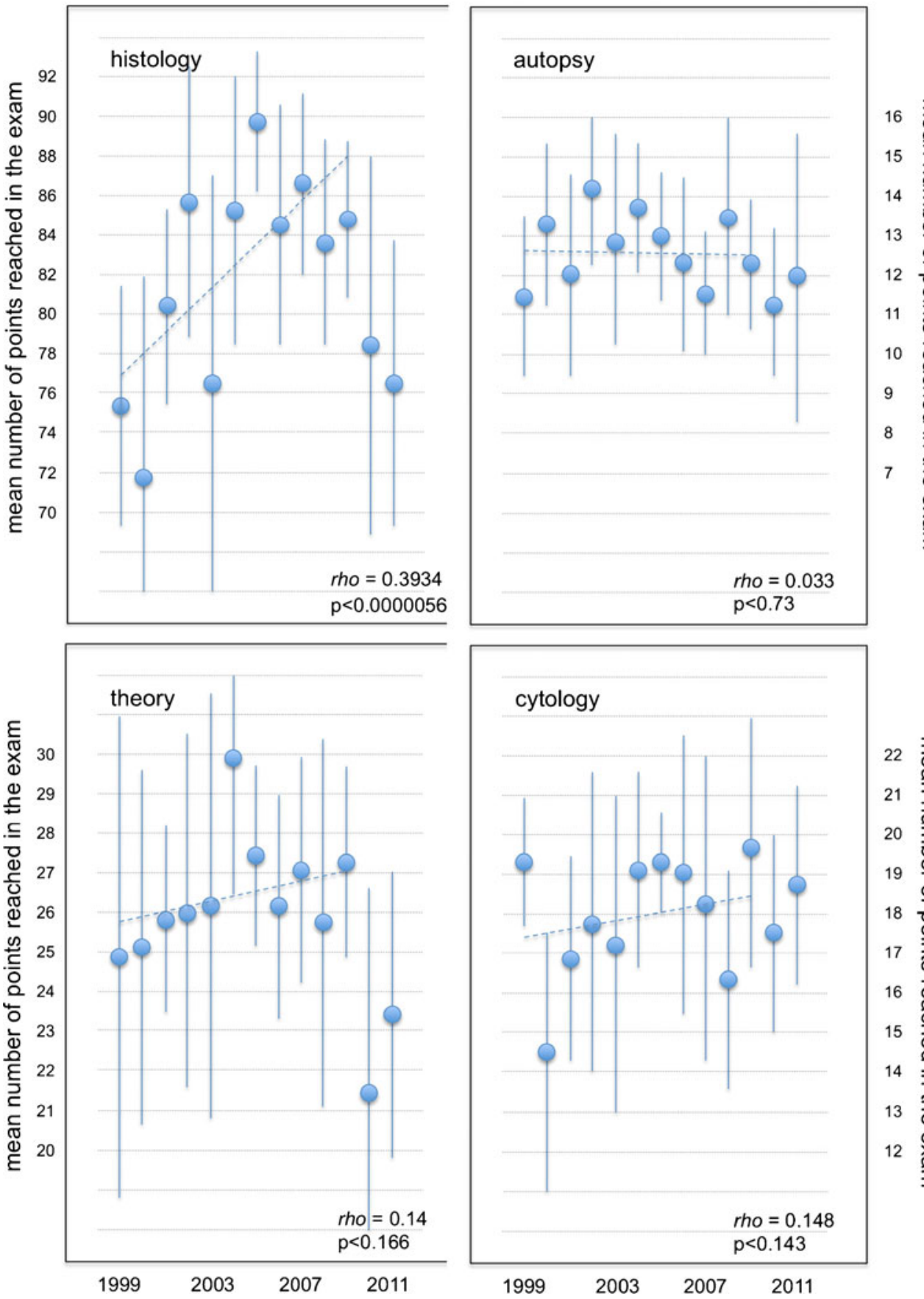

forth by the educational committee of the SSPath/SGPath. Once the revised version of the board prerequisites, in particular the (reduced) number of autopsies, as well as surgical, and cytological cases, and the (increased) number of exams in molecular pathology (FISH and PCR), is accepted by the various instances, an increasing emphasis will be placed on the examination of molecular pathology, no longer limited to questions in the theory part of the exam (knowledge), but also in the practical part of the exam (skills).

Can a 2-day exam predict and verify if the candidates who pass the exam will be good anatomical pathologists?
Certainly, it is not possible for such an exam to fully cover all aspects of the multi-faceted and complex nature of our profession. Communication skills, professional attitudes related to patient management, quality control, and error culture, as well as laboratory management, can certainly not be thoroughly tested, to name only a few examples. However, no board examination can claim to reliably test the future performance of medical professionals. In fact, Hamdy and coworkers have concluded from a thorough review of professional exams in various fields of medicine that evidence on predictors of performance in practice beyond residency training is rare 
and weak [2]. Consistent with this notion, Cox has rightly stated that testing skills in an exam can at best test the prerequisites for professional performance rather than the performance itself, which includes processes and outputs in one of many practice settings [3]. The SGPath board examination in anatomical pathology tries its best to evaluate the skills and the knowledge of our future professionals. Whether or not the exam in its present design responds to this effort remains speculative. Even though we regularly ask candidates after the exam about their opinions, their mostly positive answers are likely biased by the particular situation in which they find themselves at that very moment, having just been told that the hard work of many months has been rewarded by a piece of paper with the magic word "passed."

End of service exams of professional proficiency are not mandatory in all member countries of the European Union. Based on a survey among members of countries represented in the European Section/Board of Pathology [4], all but six countries offer similar, albeit usually shorter (usually 1 day or less) and less extensive exams (often without autopsy or cytology parts). In six countries, in particular from southern Europe and Scandinavia, no end of service exams are offered. In these countries, the responsibility to verify the proficiency of former residents resides within the program director or the peer group of past and future colleagues.

Other than fulfilling a certain moral obligation towards society to verify the capacity of future professionals in anatomical pathology, the question may indeed be asked whether there is any gain to be derived from taking the exam. Since candidates are very well aware of the fact that the examination tests their knowledge and skills related to the diagnostic routine, they typically prepare for it by going over collections of slides, either alone or in study groups with one or several colleagues, and by reviewing quite a bit more than usual the existing diagnostic literature (i.e., pathology journals and recent textbooks). This preparation may in itself be considered as a clear advantage, and some former candidates have stated that they have never been more "fit" in pathology than during the preparation for the exam.

Acknowledgments The authors of this manuscript have either functioned as presidents and/or elected members of the standing exam committee for periods of usually $4-5$ years and/or contributed in a substantial way to the exam and to this manuscript. They would like to acknowledge the excellent assistance and support by the secretaries of the presidents of the exam committee, Ghislaine Perriard and Murielle Barman in Lausanne, Doris Manca in Neuchâtel, as well as Brigitte Terrier in Zürich.

Conflict of interest Neither the presidents nor the elected members of the examination committee are paid for their activities, except for their travel costs to the exam venues, which are reimbursed by the SGPath. The price paid by the candidates for the examination is currently CHF 450.00 (reduced fee of CHF 300.00 for members of the SGPath), which is being used for travel expenses for the examiners, the rental of microscopes, and other expenses linked to the exam.

\section{References}

1. Van der Vleuten C (2000) Validity of final examinations in undergraduate medical training. BMJ 321:1217-1219

2. Hamdy H, Prasad K, Anderson MB, Scherpbier A, Williams R, Zwierstra R, Cuddihy H (2006) BEME systematic review: predictive values of measurements obtained in medical schools and future performance in medical practice. Med Teach 28:103-116

3. Cox K (2000) Examining and recording clinical performance: a critique and some recommendations. Educ Heal 13:45-52

4. Bosman FT, van den Tweel JG (2009) Unison or cacophony: postgraduate training in pathology in Europe. Virchow's Arch 454:497503 